Aporte Santiaguino 11(1), enero-junio 2018: 167-180

ISSN-L 2616-9541

\title{
Clima organizacional y desempeño laboral de los trabajadores de la Administración Local de Agua Huaraz
}

\author{
Working Environment and employee's job performance at Local \\ Administration of Water Resource in Huaraz
}

Sinthia Urbano Broncano ${ }^{1}$

\section{RESUMEN}

El objetivo de esta investigación fue evaluar el impacto del clima organizacional en la mejora del desempeño laboral en los trabajadores de una institución del Estado peruano, Administración Local de Agua Huaraz (ALA-HZ). Se aplicó el método del censo que recopiló datos de la percepción de cada trabajador en relación a las variables; mediante 02 cuestionarios y 01 escala de medición de actitudes. Se realizó el análisis estadístico inferencial bivariante utilizando la prueba estadística no paramétrica de Chi-cuadrado, mediante tablas de contingencia o tabulaciones cruzadas, para evaluar la correlación e independencia a un nivel de significancia del 5\% $(\mathrm{p}<0,05)$. Se obtuvo que en la dimensión nivel de percepción global del ambiente laboral un 54\% de trabajadores calificaron el clima organizacional como favorable. Además, la dimensión nivel de percepción del ambiente laboral en relación a la infraestructura y tecnología, refleja que $8 \%$ califica el ambiente como muy favorable. En relación al desempeño laboral, 38\% lo califica como excelente. La prueba de Chi-cuadrado arroja un valor teórico igual a 88,25 menor al valor calculado de 839,44; razón por la cual se rechazó la hipótesis nula y se aceptó la hipótesis alternativa. Se concluyó que un adecuado clima organizacional mejora el desempeño laboral en los trabajadores de la ALA-HZ.

Palabras clave: desempeño laboral; clima organizacional; institución del Estado; Huaraz.

\footnotetext{
1 Universidad Nacional Santiago Antúnez de Mayolo. Huaraz, Perú.

(C) Los autores. Este artículo es publicado por la Revista Aporte Santiaguino de la Universidad Nacional Santiago Antúnez de Mayolo. Este es un artículo de acceso abierto, distribuido bajo los términos de la Licencia Creative Commons Atribución-NoComercial-CompartirIgual 4.0 Internacional. (http://creativecommons.org/licenses/ by-nc-sa/4.0/), que permite el uso no comercial, distribución y reproducción en cualquier medio, siempre que la obra original sea debidamente citada.
} 


\begin{abstract}
The research objective was to evaluate the working environment on the improvement of the public employee's performance of the Peruvian Local Administration of Water Resource in Huaraz (ALA-HZ). The census method was applied, it compiled perception data from each employee in relation to study variables; through 02 questionnaires and 01 attitudes' scale. The bivariate inferential statistical analysis was performed using the non-parametric Chi-Square statistical test, using contingency tables or cross-tabulations, to evaluate correlation and independence with a significance level of $5 \%(p<0,05)$. In the dimension denominated perception level about working environment, $54 \%$ of employees rated the working environment as favorable. In addition, the dimension perception level in relation to infrastructure and technology reflects that $8 \%$ said the working environment was very favorable. Regarding job performance, $38 \%$ rated it as excellent. Chi-Square test shows a theoretical value equal to 88,25 which is less than the calculated value of 839,44 , that is the reason why the null hypothesis is rejected and the alternative hypothesis is accepted. It was concluded that an adequate working environment improves job performance in ALA-HZ employees.
\end{abstract}

Keywords: job performance; working environment; state institution; Huaraz.

\title{
INTRODUCCIÓN
}

En nuestros días, muchos ejecutivos de organizaciones presentan mayor y mejor disposición a la opinión y sentir de los trabajadores que forman parte de su equipo, pues saben que ante un malestar laboral se ve afectado directamente el desarrollo de la organización. El caso en estudio es la Administración Local de Agua Huaraz (ALAHZ). El tema de investigación es relevante pues permite evaluar el impacto del clima organizacional en la mejora del desempeño laboral de los trabajadores y así encaminar el planteamiento de soluciones concretas frente al mediano desarrollo del factor humano y su rol en la organización. La investigación responde a la pregunta: ¿Qué impacto tiene el clima organizacional en la mejora del desempeño laboral en los trabajadores de la ALA-HZ?

En relación al clima organizacional, se sigue el paradigma de la Teoría de Likert, la cual asevera que el comportamiento asumido por los subordinados depende directamente del comportamiento administrativo y las condiciones organizacionales que los mismos perciben, por lo tanto se afirma que la reacción estará determinada por la percepción (Brunet, 1999). Se toma como modelo para la primera dimensión lo establecido por Palma (2004) y para la segunda dimensión lo indicado por Alejandro y otros (2016). 
Con respecto al desempeño laboral, la investigación se rige por el precepto de Schermerhorn (2006, citado en Enríquez, 2014: 54) que señala que el desempeño laboral es la cantidad y calidad de los trabajos realizados por un individuo, o un grupo de ellos, en el trabajo. El desempeño, como comúnmente se dice, es el balance de las personas en el trabajo. Es una piedra angular de la productividad y debe contribuir al logro de los objetivos organizacionales. Las dimensiones de esta variable son establecidas según el modelo de Cruz (2008).

El grado de relación entre estas variables ha sido tratado en diversas investigaciones, afirmándose que:

La percepción y apreciación de los empleados con relación a los aspectos estructurales (proceso y procedimientos), las relaciones entre las personas y el ambiente físico (infraestructura y elementos de trabajo), afectan las relaciones e inciden en las reacciones del comportamiento de los empleados, tanto positiva como negativamente, y por tanto, modifican el desarrollo productivo de su trabajo y de la organización (García, 2009: 48).

Por otra parte, «La productividad es el principal problema al que se enfrenta una organización y el talento humano es una parte decisiva de la solución» (Rodas, 2013: 2). Resulta interesante la afirmación «The organizational climate should be conducive both to the employer and the employees (El clima organizacional debe ser propicio tanto al empleador como a los empleados)»(Kubendran y otros, 2013: 81). En términos generales, se aprecia que la gestión del recurso humano es un factor clave para el cumplimiento de logros institucionales.

\section{MATERIALES Y MÉTODOS}

Se trata de una investigación con enfoque cuantitativo, de diseño no experimental-transeccional y carácter correlacional-causal. La población está contituída por el total de trabajadores de la ALA-HZ, periodo 2016. El método para la elección de la muestra fue no probabilístico-intencional. Para la recolección de datos se utilizó la técnica de la encuesta escrita, a través de la escala de actitud y el cuestionario. Los 03 instrumentos cuantitativos fueron estándares, uniformizados, validados.

La escala clima laboral CL-SPC está conformada por 50 ítems. Las 5 dimensiones que mide el instrumento son: Realización personal, involucramiento, laboral, supervisión, comunicación y condiciones laborales. El cuestionario de clima organizacional - nexo ambiente físico y tecnológico consta de 19 ítems. Este instrumento realiza un análisis de los factores ambiente físico y tecnología como parte del clima organizacional.

El cuestionario desempeño laboral permitió medir el desempeño. Consta de 48 ítems. 


\section{RESULTADOS}

Se empleó la estadística descriptiva con referencia a los datos alcanzados en la primera parte de la encuesta. El resultado obtenido se muestra en la tabla 1.

Tabla 1. Características de la muestra de trabajadores de la ALA-HZ

\begin{tabular}{|c|c|c|c|}
\hline & Características & Frecuencia & Porcentaje \\
\hline \multirow{3}{*}{ Edad } & $31-40$ & 7 & 54,00 \\
\hline & $41-50$ & 2 & 15,00 \\
\hline & 51 a más & 4 & 31,00 \\
\hline \multirow[b]{2}{*}{ Sexo } & Mujer & 4 & 31,00 \\
\hline & Varón & 9 & 69,00 \\
\hline \multirow{4}{*}{ Nivel Académico } & Educación básica & 3 & 23,00 \\
\hline & Educación superior no univer- & 2 & 15,00 \\
\hline & sitana & 8 & 62,00 \\
\hline & $\begin{array}{l}\text { Educación superior universi- } \\
\text { taria }\end{array}$ & & \\
\hline \multirow{5}{*}{ Tiempo de Servicio } & $<1$ año & 2 & 15,00 \\
\hline & 1 año & 1 & 8,00 \\
\hline & 2 años & 2 & 15,00 \\
\hline & 3 años & 1 & 8,00 \\
\hline & 4 años & 7 & 54,00 \\
\hline \multirow{3}{*}{ Tipo de Contrato } & CAS ALA Huaraz & 10 & 77,00 \\
\hline & CAS Rotado AAA IV HCH & 1 & 8,00 \\
\hline & Locación de servicios & 2 & 15,00 \\
\hline
\end{tabular}

Clima organizacional: El resultado de la dimensión nivel de percepción global del ambiente laboral se presenta en la tabla 2 y figura 1. 
Tabla 2. Resultados sobre cada factor del clima organizacional de los trabajadores

\begin{tabular}{lcccccccccc}
\hline & \multicolumn{1}{c}{$\begin{array}{c}\text { Muy } \\
\text { favorable }\end{array}$} & Favorable & Medio & \multicolumn{2}{c}{ Desfavora- } \\
\multicolumn{1}{c}{ Factores } & $\mathrm{F}$ & $\%$ & $\mathrm{~F}$ & $\%$ & $\mathrm{~F}$ & $\%$ & $\mathrm{~F}$ & $\%$ & \multicolumn{2}{c}{$\begin{array}{c}\text { Muy } \\
\text { desfavorable }\end{array}$} \\
\hline Autorrealización & 0 & 0 & 5 & 34,46 & 6 & 46,15 & 2 & 15,38 & 0 & 0 \\
Involucramiento laboral & 1 & 7,69 & 11 & 84,62 & 1 & 7,69 & 0 & 0 & 0 & 0 \\
Supervisión & 0 & 0 & 9 & 69,23 & 3 & 23,08 & 1 & 7,69 & 0 & 0 \\
Comunicación & 0 & 0 & 9 & 69,23 & 3 & 23,08 & 1 & 7,69 & 0 & 0 \\
Condiciones laborales & 0 & 0 & 6 & 46,15 & 7 & 53,85 & 0 & 0 & 0 & 0 \\
\hline
\end{tabular}

En el análisis de los resultados se encontró que un 54\% de ellos calificaron el clima como favorable y el $46 \%$ como medio.

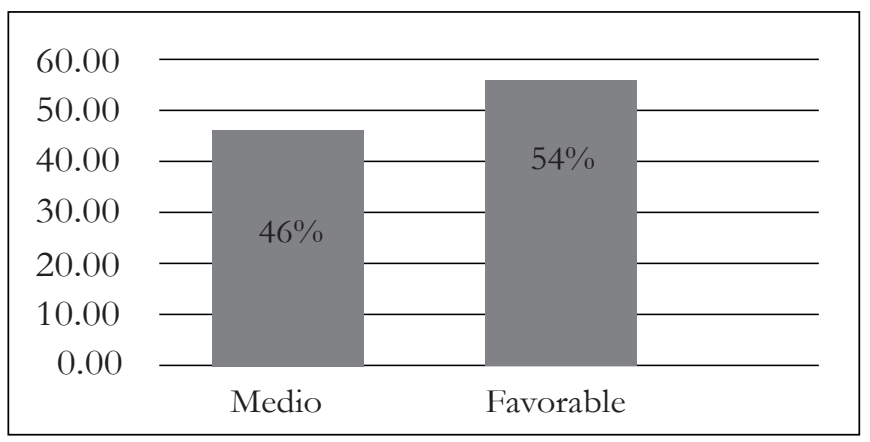

Figura 1. Distribución de porcentajes para el clima organizacional

Clima organizacional: El resultado de la dimensión nivel de percepción del ambiente laboral en relación a la tecnología y ambiente físico se presenta en la tabla 3 y figura 2 .

Tabla 3. Resultados sobre cada factor del clima organizacional - tecnología y ambiente físico

\begin{tabular}{lcccccccccc}
\hline & \multicolumn{1}{c}{$\begin{array}{c}\text { Muy } \\
\text { favorable }\end{array}$} & \multicolumn{1}{c}{ Favorable } & Medio & \multicolumn{2}{c}{ Desfavorable } & \multicolumn{2}{c}{$\begin{array}{c}\text { Muy } \\
\text { desfavorable }\end{array}$} \\
Factores & $\mathrm{F}$ & $\%$ & $\mathrm{~F}$ & $\%$ & $\mathrm{~F}$ & $\%$ & $\mathrm{~F}$ & $\%$ & $\mathrm{~F}$ & $\%$ \\
\hline Tecnología & 4 & 30,77 & 8 & 61,54 & 1 & 7,69 & 0 & 0 & 0 & 0 \\
Ambiente Físico & 0 & 0 & 12 & 92,31 & 1 & 7,69 & 0 & 0 & 0 & 0 \\
\hline
\end{tabular}

Para esta dimensión se encontró que un $84 \%$ de los trabajadores calificaron el clima como favorable, $8 \%$ como muy favorable y otro $8 \%$ como medio. 


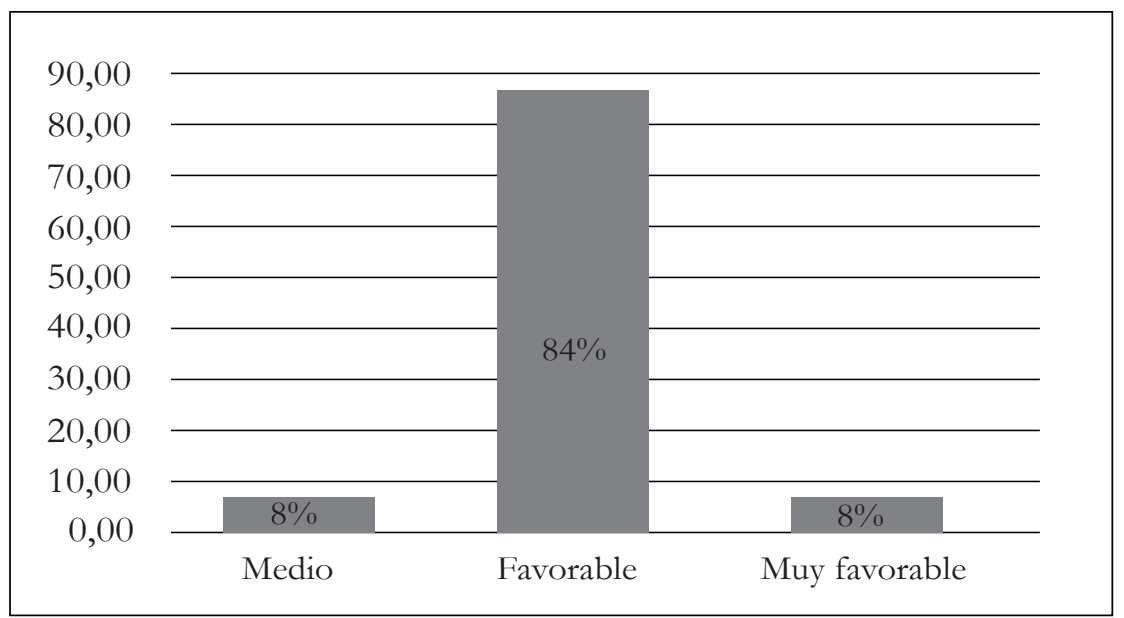

Figura 2. Distribución de porcentajes para el clima organizacional -tecnología y ambiente físico

Desempeño laboral de los trabajadores: El detalle del procesamiento en relación a esta variable se muestra en la tabla 4 y figura 3.

Tabla 4. Análisis de resultados sobre el desempeño laboral de los trabajadores

\begin{tabular}{ccc}
\hline Categoría & Frecuencia & Porcentaje \\
\hline Medio & 3 & 23,10 \\
Favorable & 8 & 61,50 \\
Muy favorable & 2 & 15,40 \\
Total & 13 & 100,00 \\
\hline
\end{tabular}

Se halló que un 62\% de trabajadores lo calificaron como favorable, 15\% como muy favorable y $23 \%$ como medio.

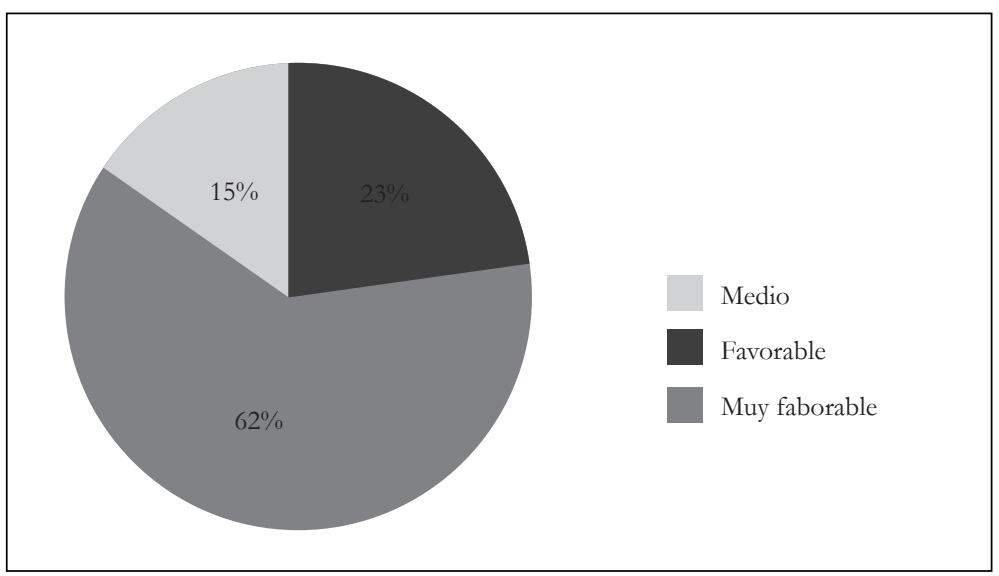


Figura 3. Distribución de porcentajes para el desempeño laboral

Prueba de hipótesis

Empleando la estadística inferencial, se realizó la prueba de la hipótesis general.

Formulación o modelo lógico:

$\mathrm{H}_{\mathrm{o}}$ : “Un adecuado clima organizacional NO mejora el desempeño laboral en los trabajadores de la Administración Local de Agua Huaraz, periodo 2016."

$\mathrm{H}_{1}$ : "Un adecuado clima organizacional mejora el desempeño laboral en los trabajadores de la Administración Local de Agua Huaraz, periodo 2016.”

Modelo matemático:

$\mathrm{H}_{\mathrm{o}}: \mathrm{f}_{\mathrm{ij}}=\mathrm{e}_{\mathrm{ij}} ; \mathrm{H}_{1}: \mathrm{f}_{\mathrm{ij}} \neq \mathrm{e}_{\mathrm{ij}}$

$$
x^{2} \text { calc }=\sum_{i} \sum_{j} \frac{\left(f_{i j-} e_{i j}\right)^{2}}{e_{i j}}
$$

Terminología:

$\mathrm{H}_{\mathrm{o}}$ : Hipótesis nula, $\mathrm{H}_{1}$ : Hipótesis alternativa, $\chi_{\text {calc }}^{2}$ : Chi-cuadrado calculado, $\mathrm{f}_{\mathrm{ij}}$ : Frecuencias observadas y e ${ }_{i j}$ : Frecuencias esperadas.

Nivel de significación:

Considerando un nivel de confianza del 95\% se trabaja con un nivel de significancia del $5 \%$.

Prueba estadística no Paramétrica de Chi-cuadrado:

Fórmula para calcular los grados de libertad

G.D.L $=\left(\mathrm{N}^{\circ}\right.$ FILAS -1$) \times\left(\mathrm{N}^{\circ}\right.$ COLUMNAS -1)

G.D.L $=(18-1) \times(5-1)=68$

Al trabajar con un nivel de significancia del 0.05. El valor del Chi-cuadrado teórico para 68 grados de libertad es 88,25 .

Aplicando la fórmula se obtiene el valor de Chi-cuadrado calculado:

$$
\chi_{\text {calc }}^{2}=\sum_{i} \sum_{j} \frac{\left(f_{i j}-e_{i j}\right)^{2}}{e_{i j}}=839,44
$$


Tabla 5. Cálculo de Chi-cuadrado por factores de las variables (hipótesis general)

\begin{tabular}{|c|c|c|c|c|c|c|c|}
\hline \multirow{2}{*}{\multicolumn{2}{|c|}{ Factor }} & \multicolumn{5}{|c|}{ Alternativa } & \multirow{2}{*}{ Total } \\
\hline & & & & & & & \\
\hline \multirow{5}{*}{$\begin{array}{l}\text { Autorrealiza- } \\
\text { ción }\end{array}$} & Observed & 4 & 32 & 42 & 44 & 8 & 130 \\
\hline & Expected & 2,17 & 11,01 & 26,12 & 41,32 & 49,38 & 130,00 \\
\hline & $O-E$ & 1,83 & 20,99 & 15,88 & 2,68 & $-41,38$ & 0,00 \\
\hline & $(\mathrm{O}-\mathrm{E})^{2} / \mathrm{E}$ & 1,54 & 40,03 & 9,65 & 0,17 & 34,68 & 86,07 \\
\hline & $\%$ Of Chisq & $0,2 \%$ & $4,8 \%$ & $1,1 \%$ & $0,0 \%$ & $4,1 \%$ & $10,3 \%$ \\
\hline \multirow{5}{*}{$\begin{array}{l}\text { Involucra- } \\
\text { miento labo- } \\
\text { ral }\end{array}$} & Observed & 0 & 9 & 33 & 74 & 14 & 130 \\
\hline & Expected & 2,17 & 11,01 & 26,12 & 41,32 & 49,38 & 130,00 \\
\hline & $\mathrm{O}-\mathrm{E}$ & $-2,17$ & $-2,01$ & 6,88 & 32,68 & $-35,38$ & 0,00 \\
\hline & $(\mathrm{O}-\mathrm{E})^{2} / \mathrm{E}$ & 2,17 & 0,37 & 1,81 & 25,85 & 25,35 & 55,55 \\
\hline & $\%$ Of Chisq & $0,3 \%$ & $0,0 \%$ & $0,2 \%$ & $3,1 \%$ & $3,0 \%$ & $6,6 \%$ \\
\hline \multirow[t]{5}{*}{ Supervisión } & Observed & 3 & 22 & 41 & 53 & 11 & 130 \\
\hline & Expected & 2,17 & 11,01 & 26,12 & 41,32 & 49,38 & 130,00 \\
\hline & $O-E$ & 0,83 & 10,99 & 14,88 & 11,68 & $-38,38$ & 0,00 \\
\hline & $(\mathrm{O}-\mathrm{E})^{2} / \mathrm{E}$ & 0,32 & 10,98 & 8,47 & 3,30 & 29,83 & 52,90 \\
\hline & $\%$ Of Chisq & $0,0 \%$ & $1,3 \%$ & $1,0 \%$ & $0,4 \%$ & $3,6 \%$ & $6,3 \%$ \\
\hline \multirow{5}{*}{$\begin{array}{l}\text { Comunica- } \\
\text { ción }\end{array}$} & Observed & 1 & 15 & 52 & 49 & 13 & 130 \\
\hline & Expected & 2,17 & 11,01 & 26,12 & 41,32 & 49,38 & 130,00 \\
\hline & $O-E$ & $-1,17$ & 3,99 & 25,88 & 7,68 & $-36,38$ & 0,00 \\
\hline & $(\mathrm{O}-\mathrm{E})^{2} / \mathrm{E}$ & 0,63 & 1,45 & 25,63 & 1,43 & 26,80 & 55,94 \\
\hline & $\%$ Of Chisq & $0,1 \%$ & $0,2 \%$ & $3,1 \%$ & $0,2 \%$ & $3,2 \%$ & $6,7 \%$ \\
\hline \multirow{5}{*}{$\begin{array}{l}\text { Condiciones } \\
\text { laborales }\end{array}$} & Observed & 6 & 26 & 39 & 45 & 14 & 130 \\
\hline & Expected & 2,17 & 11,01 & 26,12 & 41,32 & 49,38 & 130,00 \\
\hline & $O-E$ & 3,83 & 14,99 & 12,88 & 3,68 & $-35,38$ & 0,00 \\
\hline & $(\mathrm{O}-\mathrm{E})^{2} / \mathrm{E}$ & 6,76 & 20,42 & 6,35 & 0,33 & 25,35 & 59,20 \\
\hline & $\%$ Of Chisq & $0,8 \%$ & $2,4 \%$ & $0,8 \%$ & $0,0 \%$ & $3,0 \%$ & $7,1 \%$ \\
\hline \multirow[t]{5}{*}{ Tecnología } & Observed & 0 & 7 & 50 & 52 & 21 & 130 \\
\hline & Expected & 2,17 & 11,01 & 26,12 & 41,32 & 49,38 & 130,00 \\
\hline & $O-E$ & $-2,17$ & $-4,01$ & 23,88 & 10,68 & $-28,38$ & 0,00 \\
\hline & $(\mathrm{O}-\mathrm{E})^{2} / \mathrm{E}$ & 2,17 & 1,46 & 21,82 & 2,76 & 16,31 & 44,52 \\
\hline & \% Of Chisq & $0,3 \%$ & $0,2 \%$ & $2,6 \%$ & $0,3 \%$ & $1,9 \%$ & $5,3 \%$ \\
\hline \multirow{5}{*}{$\begin{array}{l}\text { Ambiente } \\
\text { físico }\end{array}$} & Observed & 2 & 15 & 45 & 43 & 12 & 117 \\
\hline & Expected & 1,95 & 9,91 & 23,51 & 37,19 & 44,44 & 117,00 \\
\hline & $O-E$ & 0,05 & 5,09 & 21,49 & 5,81 & $-32,44$ & 0,00 \\
\hline & $(\mathrm{O}-\mathrm{E})^{2} / \mathrm{E}$ & 0,00 & 2,62 & 19,64 & 0,91 & 23,68 & 46,85 \\
\hline & $\%$ Of Chisq & $0,0 \%$ & $0,3 \%$ & $2,3 \%$ & $0,1 \%$ & $2,8 \%$ & $5,6 \%$ \\
\hline
\end{tabular}




\begin{tabular}{|c|c|c|c|c|c|c|c|}
\hline \multirow{2}{*}{\multicolumn{2}{|c|}{ Factor }} & \multicolumn{5}{|c|}{ Alternativa } & \multirow{2}{*}{ Total } \\
\hline & & & Cas & Regu- & Casi & & \\
\hline \multirow[t]{5}{*}{ Planeación } & Observed & 1 & 4 & 3 & 7 & 11 & 26 \\
\hline & Expected & 0,43 & 2,20 & 5,22 & 8,26 & 9,88 & 26,00 \\
\hline & $\mathrm{O}-\mathrm{E}$ & 0,57 & 1,80 & $-2,22$ & $-1,26$ & 1,12 & 0,00 \\
\hline & $(\mathrm{O}-\mathrm{E})^{2} / \mathrm{E}$ & 0,74 & 1,47 & 0,95 & 0,19 & 0,13 & 3,48 \\
\hline & $\%$ Of Chisq & $0,1 \%$ & $0,2 \%$ & $0,1 \%$ & $0,0 \%$ & $0,0 \%$ & $0,4 \%$ \\
\hline \multirow[t]{5}{*}{ Organización } & Observed & 2 & 4 & 2 & 6 & 12 & 26 \\
\hline & Expected & 0,43 & 2,20 & 5,22 & 8,26 & 9,88 & 26,00 \\
\hline & $\mathrm{O}-\mathrm{E}$ & 1,57 & 1,80 & $-3,22$ & $-2,26$ & 2,12 & 0,00 \\
\hline & $(\mathrm{O}-\mathrm{E})^{2} / \mathrm{E}$ & 5,65 & 1,47 & 1,99 & 0,62 & 0,46 & 10,18 \\
\hline & $\%$ Of Chisq & $0,7 \%$ & $0,2 \%$ & $0,2 \%$ & $0,1 \%$ & $0,1 \%$ & $1,2 \%$ \\
\hline \multirow{5}{*}{$\begin{array}{l}\text { Dirección/ } \\
\text { Ejecución }\end{array}$} & Observed & 1 & 1 & 7 & 10 & 33 & 52 \\
\hline & Expected & 0,87 & 4,40 & 10,45 & 16,53 & 19,75 & 52,00 \\
\hline & $\mathrm{O}-\mathrm{E}$ & 0,13 & $-3,40$ & $-3,45$ & $-6,53$ & 13,25 & 0,00 \\
\hline & $(\mathrm{O}-\mathrm{E})^{2} / \mathrm{E}$ & 0,02 & 2,63 & 1,14 & 2,58 & 8,89 & 15,25 \\
\hline & $\%$ Of Chisq & $0,0 \%$ & $0,3 \%$ & $0,1 \%$ & $0,3 \%$ & $1,1 \%$ & $1,8 \%$ \\
\hline \multirow[t]{5}{*}{ Control } & Observed & 1 & 2 & 3 & 5 & 15 & 26 \\
\hline & Expected & 0,43 & 2,20 & 5,22 & 8,26 & 9,88 & 26,00 \\
\hline & $\mathrm{O}-\mathrm{E}$ & 0,57 & $-0,20$ & $-2,22$ & $-3,26$ & 5,12 & 0,00 \\
\hline & $(\mathrm{O}-\mathrm{E})^{2} / \mathrm{E}$ & 0,74 & 0,02 & 0,95 & 1,29 & 2,66 & 5,65 \\
\hline & $\%$ Of Chisq & $0,1 \%$ & $0,0 \%$ & $0,1 \%$ & $0,2 \%$ & $0,3 \%$ & $0,7 \%$ \\
\hline \multirow{5}{*}{$\begin{array}{l}\text { Relación con } \\
\text { los superiores }\end{array}$} & Observed & 0 & 4 & 3 & 8 & 24 & 39 \\
\hline & Expected & 0,65 & 3,30 & 7,84 & 12,40 & 14,81 & 39,00 \\
\hline & $\mathrm{O}-\mathrm{E}$ & $-0,65$ & 0,70 & $-4,84$ & $-4,40$ & 9,19 & 0,00 \\
\hline & $(\mathrm{O}-\mathrm{E})^{2} / \mathrm{E}$ & 0,65 & 0,15 & 2,99 & 1,56 & 5,70 & 11,04 \\
\hline & $\%$ Of Chisq & $0,1 \%$ & $0,0 \%$ & $0,4 \%$ & $0,2 \%$ & $0,7 \%$ & $1,3 \%$ \\
\hline \multirow{5}{*}{$\begin{array}{l}\text { Relación con } \\
\text { los compañe- } \\
\text { ros }\end{array}$} & Observed & 0 & 0 & 2 & 11 & 26 & 39 \\
\hline & Expected & 0,65 & 3,30 & 7,84 & 12,40 & 14,81 & 39,00 \\
\hline & $\mathrm{O}-\mathrm{E}$ & $-0,65$ & $-3,30$ & $-5,84$ & $-1,40$ & 11,19 & 0,00 \\
\hline & $(\mathrm{O}-\mathrm{E})^{2} / \mathrm{E}$ & 0,65 & 3,30 & 4,35 & 0,16 & 8,45 & 16,90 \\
\hline & $\%$ Of Chisq & $0,1 \%$ & $0,4 \%$ & $0,5 \%$ & $0,0 \%$ & $1,0 \%$ & $2,0 \%$ \\
\hline \multirow{5}{*}{$\begin{array}{l}\text { Relación con } \\
\text { el público }\end{array}$} & Observed & 0 & 0 & 0 & 11 & 41 & 52 \\
\hline & Expected & 0,87 & 4,40 & 10,45 & 16,53 & 19,75 & 52,00 \\
\hline & $O-E$ & $-0,87$ & $-4,40$ & $-10,45$ & $-5,53$ & 21,25 & 0,00 \\
\hline & $(\mathrm{O}-\mathrm{E})^{2} / \mathrm{E}$ & 0,87 & 4,40 & 10,45 & 1,85 & 22,86 & 40,43 \\
\hline & $\%$ Of Chisq & $0,1 \%$ & $0,5 \%$ & $1,2 \%$ & $0,2 \%$ & $2,7 \%$ & $4,8 \%$ \\
\hline
\end{tabular}




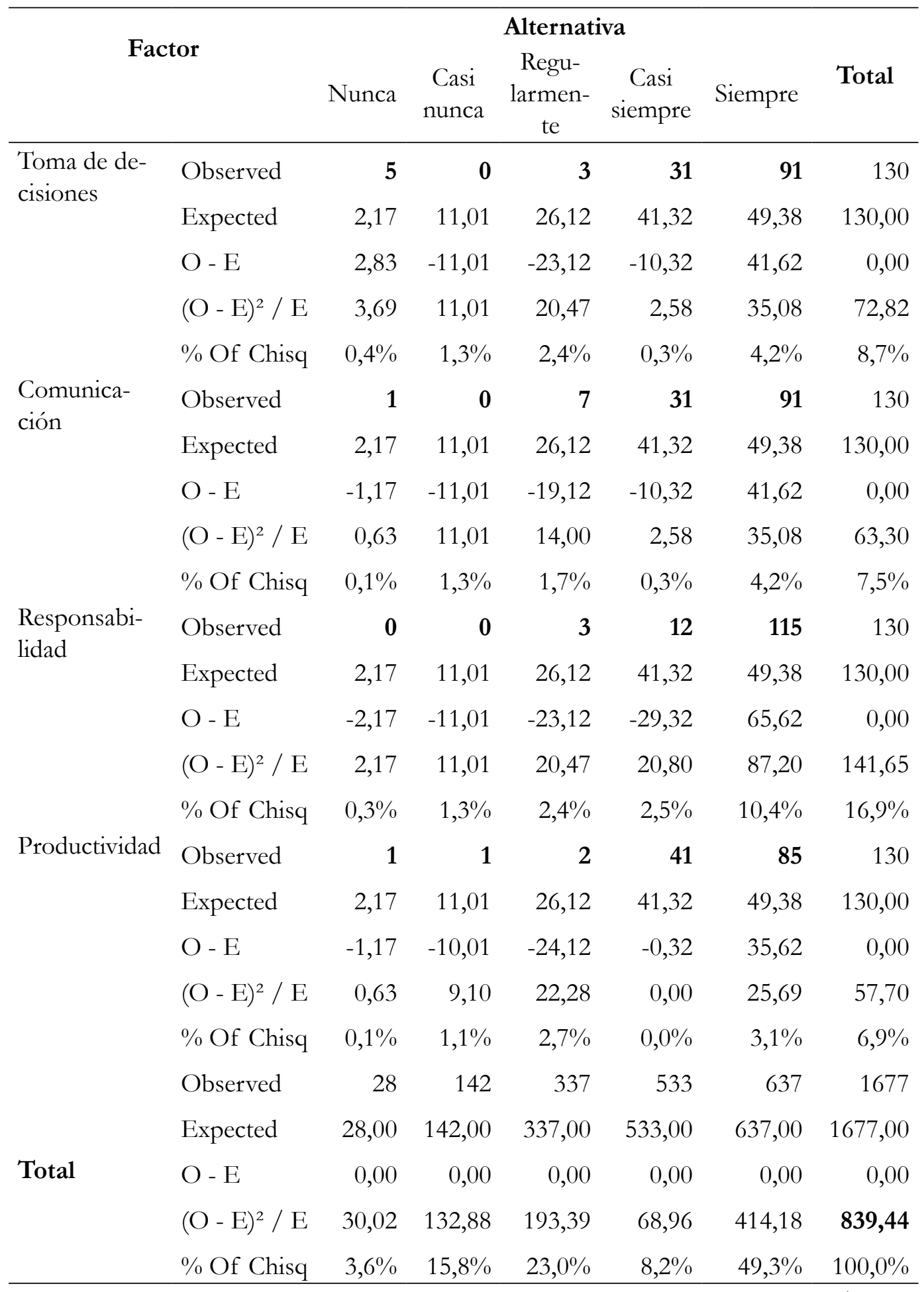

Nota: Observed, son los datos observados; Expected, son los datos esperados; $(\mathrm{O}-\mathrm{E})^{2} / \mathrm{E}$ es el valor de Chi-cuadrado. 
Regla de decisión: Dado que Chi-cuadrado teórico < Chi-cuadrado calculado.

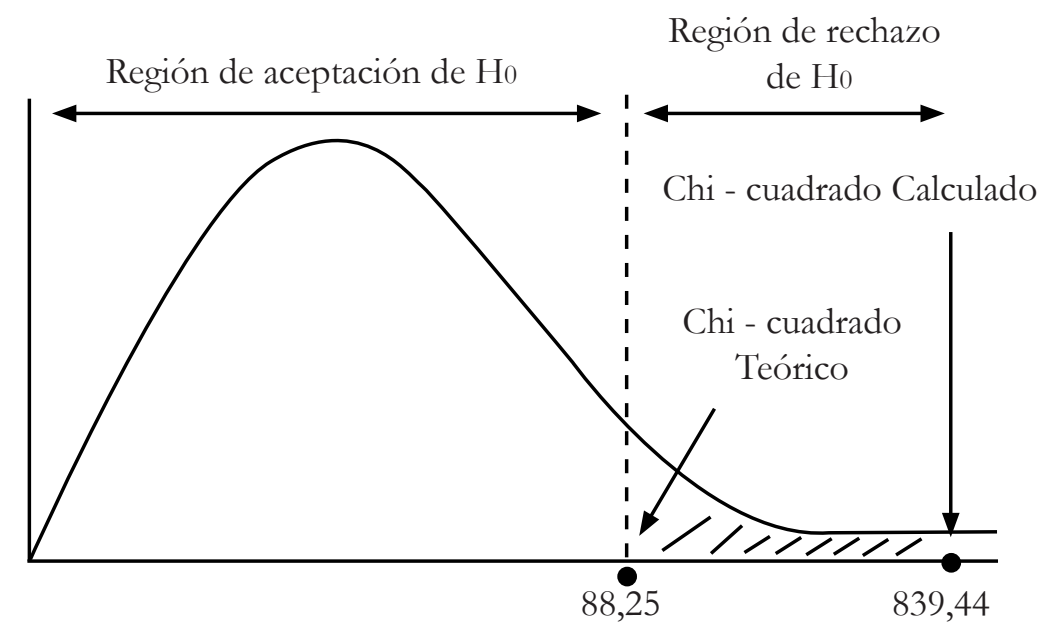

Figura 4. Comprobación de hipótesis general

Se rechaza la hipótesis nula y se acepta o refuerza la hipótesis alternativa " $\mathrm{H}_{1}$ ".

\section{DISCUSIÓN}

El clima organizacional en su dimensión nivel de percepción global de ambiente laboral, mostró el predominio de un clima organizacional del tipo favorable (54\%), seguido del clima medio (46\%). Estos resultados son semejantes a los encontrados por Pérez y Rivera (2015), pero discordante con lo hallado por Beltrán y Palomino (2014).

De otro lado, el clima organizacional en su dimensión en relación a la tecnología y ambiente físico, señala que un $84 \%$ de los trabajadores calificaron el clima como favorable, $8 \%$ como muy favorable y otro $8 \%$ como medio. Estos resultados son semejantes al postulado de García (2009); no obstante discordante con Alejandro y otros (2016).

Al analizar el desempeño laboral observamos que en los trabajadores de la Administración Local de Agua Huaraz, predomina un desempeño del tipo favorable (62\%), seguido de tipo medio (23\%) y muy favorable (15\%). Resaltan indicadores como la ejecución de tareas de acuerdo a lo planeado y con agrado, respetando las indicaciones de los superiores, brindando un trato amable al público sin importar su clase social ni religión, siendo productivos aunque se esté sometido a estrés. Estos resultados son muy similares a los encontrados por Marroquín y Pérez (2011). 
Cuando se realizó el análisis para contrastar la hipótesis planteada, teniendo en cuenta el objetivo general y problema planteado, a través de la prueba de Chi-cuadrado resalta que el resultado es estadísticamente significativos $(\mathrm{p}<0,05)$, además de que el valor teórico de la prueba $(88,25)$ es menor al valor calculado de Chi-cuadrado $(839,44)$; por lo que se puede afirmar que existe correspondencia entre el clima organizacional y el desempeño laboral. Mientras mejor sea la percepción del clima organizacional, mayor será el desempeño de los trabajadores de la institución. Se valida y refuerza la hipótesis general. Se encontró que cuando el clima es alto también se presenta un alto desempeño laboral en el 62\% de los trabajadores de la ALA-HZ. Contrariamente, cuando se presenta un nivel medio del clima laboral la totalidad de trabajadores en este grupo calificó al desempeño laboral como bajo. Este resultado es interesante, ya que va de la mano con los hallazgos encontrados en las investigaciones a nivel internacional de Pérez (2014) y Rodríguez y otros (2011); así también, con las investigaciones nacionales de Guevara y Tafur (2015) y Mino (2014); sin embargo resulta ser discordante a lo hallado por Meléndez (2015). Así mismo, el resultado de la investigación es coherente con los postulados presentados por Kubendran y otros (2013), García (2009), Rodríguez (1992, citado en Pérez, 2014), Brow (1990, citado en Prado, 2015) y Dessler (1993, citado en Prado, 2015); pues señalan que el clima influye en el rendimiento de los colaboradores.

\section{CONCLUSIONES}

Sobre la base de los hallazgos encontrados, existe relación directa y significativa entre el clima organizacional y el desempeño laboral en los trabajadores de la Administración Local de Agua Huaraz. Este resultado prueba la validez de la hipótesis general de la investigación y permite fortalecer los hallazgos de los estudios realizados sobre la temática. Así mismo, se puede afirmar que, mientras mejor sea la percepción del clima organizacional, mayor será el desempeño de los colaboradores de la institución estatal.

Si bien es cierto los trabajadores están satisfechos con su trabajo, existen factores como: la remuneración, tecnología que no facilita el trabajo, la difícil comunicación con el superior, la falta de reconocimiento, el régimen laboral (CAS y Locación de servicios) al cual pertenecen gran parte de los trabajadores, la poca posibilidad de crecimiento profesional, la falta de capacitaciones continuas y promoción del desarrollo personal, el trato injusto, la poca innovación en la atención y procesos etc. Estos factores influyen en los trabajadores y hacen que no se sientan plenamente satisfechos y motivados. 


\section{REFERENCIAS BIBLIOGRÁFICAS}

Alejandro, Eduardo; Chávez, Rubén y Palomo, Sergio. 2016. «Ambiente Físico y Tecnología, Componentes del Clima Organizacional: Percepción a Nivel de Facultad de una Universidad en México». Revista Global de Negocios. Vol. 4, No 5. México: Universidad Autónoma de Zacatecas. 1-13.

Beltrán, Enrique y Palomino, Melba. 2014. Propuesta para mejorar la satisfacción laboral en una institución educativa a partir de la gestión del clima laboral. Escuela de Postgrado. Lima: Universidad del Pacífico.

Cruz, Denisse. 2008. Autopercepción de la Capacitación y del Desempeño Laboral de los empleados del Hospital La Carlota en Montemorelos, Nuevo León. Maestría en Administración. Facultad de Ciencias Administrativas. México: Universidad de Montemorelos.

Enríquez, Patricia. 2014. Motivación y Desempeño Laboral de los Empleados del Instituto de La Visión en México. Tesis de Posgrado. México: Universidad de Montemorelos.

García, Mónica. 2009. «Clima Organizacional y su Diagnóstico: Una aproximación Conceptual.». En Cuadernos de Administración. Cali: Universidad del Valle Sede San Fernando. 42-61.

Guevara, Franklin y Tafur, Andersson. 2015. Influencia del Clima Laboral en el Desempeño de los trabajadores de la empresa Kentucky Fried Chicken Sede Real Plaza en la Ciudad de Trujillo 2015. Facultad de Ciencias Económicas. Trujillo: Universidad Privada Antenor Orrego.

Kubendran, V.; Sampath, M. y Muthukumar, S. 2013. «Organisational climate's impact on employee's performance.» Indian Journal of Economics and Development. Vol. 1, $\mathrm{N}^{\mathrm{o}}$ 3. India: Bharathiar University. 76-81. < http:/ ijed.informaticspublishing. com/index.php/ijed/article/viewFile/33621/27748>. [Consulta: 29-08-2017].

Marroquín, Stefani y Pérez, Lorena. 2011. El clima organizacional y su relación con el desempeño laboral en los trabajadores de Burger King. Guatemala: Universidad de San Carlos de Guatemala.

Meléndez, Raúl. 2015. Relación entre el clima laboral y el desempeño de los servidores de la subsecretaría administrativa financiera del ministerio de finanzas, en el periodo 2013-2014. Maestría en Gestión del Talento Humano. Quito: Universidad Tecnológica Equinoccial.

Mino, Edgardo. 2014. Correlación entre el Clima Organizacional y el Desempeño en los Trabajadores del Restaurante de Parrillas Marakos 490 del Departamento de Lambayeque. Chiclayo: Universidad Católica Santo Toribio de Mogrovejo.

Palma, Sonia. 2004. Escala Clima Laboral CL - SPC. Manual. Lima, Perú: Cartolan E.I.R.L. 
Pérez, Fausto. 2014. El Clima Organizacional y su incidencia en el desempeño laboral de los trabajadores del MIES (Dirección Provincial Pichincha). Quito: Universidad Central del Ecuador.

Pérez, Néstor y Rivera, Pedro. 2015. Clima organizacional y satisfacción laboral en los trabajadores del instituto de investigaciones de la Amazonía Peruana, periodo 2013. Facultad de Ciencias Económicas y de Negocios. Iquitos: Universidad Nacional de la Amazonía Peruana.

Prado, Cynttia. 2015. Relación entre clima laboral y desempeño laboral en los trabajadores administrativos de la Universidad Cesar Vallejo De Trujillo. Sección de Postgrado en Ingeniería. Trujillo: Universidad Nacional de Trujillo.

Rodas, Karla. 2013. Modelo de desarrollo de clima organizacional para la Corporación Nacional de Telecomunicaciones CNT Empresa Pública de la provincia del Cañar. Ibarra: Universidad Técnica del Norte.

Rodríguez, Andrés y otros. 2011. «Clima y Satisfacción Laboral como Predictores del Desempeño: En una Organización Estatal Chilena». Saludy Sociedad. Vol. 2, N ${ }^{\circ}$ 2. Chile: Universidad Andrés Bello. 219-234.

Recepción: 03/04/2018

Aceptación: 03/05/2018

\section{Correspondencia}

Sinthia Vanesa Urbano Broncano

siva_2511@hotmail.com 